\title{
KOOPERATIVER BERICHT ÜBER \#VBIB20 - DIE VIRTUELLE KONFERENZ VON BIB UND TIB RUND UM BIBLIOTHEKARISCHE THEMEN (26.-28. MAI 2020)
}

\section{von Harald Albrecht, Bruno Bauer, Susanne Blumesberger, Elisabeth Erasimus, Daniel Formanek und Caroline Reitbrecht}

Zusammenfassung: Nach der Covid-19-bedingten Absage des 109. Deutschen Bibliothekartags, der von 26. bis 29. Mai 2020 in Hannover stattfinden hätte sollen, wurde vom Berufsverband Information Bibliothek (BIB) und von TIB - Leibniz-Informationszentrum Technik und Naturwissenschaften und Universitätsbibliothek mit \#VBIB20 zwischen 26. und 28. Mai 2020 kurzfristig eine virtuelle Konferenz rund um bibliothekarische Themen organisiert. Im vorliegenden kooperativen Bericht werden Vorträge zu den Themenbereichen Publikationswesen, Open Access, Forschungsdatenmanagement, Bibliotheksstatistik und NS-Provenienzforschung vorgestellt.

Schlagwörter: 109. Deutscher Bibliothekartag; Hannover 2020; Absage; \#vBIB20; virtuelle Konferenz; kooperativer Bericht

\section{COOPERATIVE REPORT OF \#VBIB20 - THE VIRTUAL CONFERENCE OF BIB AND TIB ABOUT LIBRARY TOPICS (MAY 26-28, 2020)}

Abstract: After the cancellation of the 109. Deutscher Bibiothekartag (109 ${ }^{\text {th }}$ German Librarian Day), which should have taken place in Hannover from Mai $26^{\text {th }}$ to $29^{\text {th }}$ 2020, the Berufsverband Information Bibliothek (BIB) and the TIB - Leibniz Information Centre for Science and Technology University Library - together with \#vBIB20 organised at short notice a virtual conference about library topics from May $26^{\text {th }}$ to $28^{\text {th }} 2020$. In this cooperative report lectures are presented about publication system, Open Access, research data management, library statistics and provenance research.

Keywords: $109^{\text {th }}$ German Librarian Day; Hannover 2020; cancellation; \#vBIB20; virtual conference; cooperative report

DOI: https://doi.org/10.31263/voebm.v73i2.4018

(c) Harald Albrecht, Bruno Bauer, Susanne Blumesberger, Elisabeth Erasimus, Daniel Formanek, Caroline Reitbrecht 


\section{Inhalt}

- \#vBIB20 - die virtuelle Konferenz rund um bibliothekarische Themen (Bruno Bauer)

- Quo vadis Peer-Review? (Daniel Formanek)

- Zurückgezogene Artikel in wissenschaftlichen Zeitschriften (Retractions): Ein Thema für wissenschaftliche Bibliotheken?! (Caroline Reitbrecht)

- DeepGreen im Testbetrieb: Erkenntnisse und Schlussfolgerungen (Caroline Reitbrecht)

- Kostentransparenz in OA-Transformationsverträgen (Daniel Formanek)

- Die neuen Personalkennzahlen in der Deutschen Bibliotheksstatistik - Neue Perspektiven für den Leistungsvergleich? (Elisabeth Erasimus)

- Bibliotheksstatistik: Wie die Visitzählung in der DBS funktioniert und wie sie den Blick auf Bibliotheken verändert (Elisabeth Erasimus)

- Forschungsdatenmanagement gestern, heute und morgen zwischen FAIR, CARE und EOSC. Ein Erfahrungsbericht mit einem digitalen Vortrag im Rahmen der virtuellen Konferenz \#vBIB20 (Susanne Blumesberger)

- Resümee aus der Sicht eines Teilnehmers der virtuellen Konferenz \#vBIB20 (Daniel Formanek)

- Provenienzforschung in der ZBW: Erste Ergebnisse zu verdächtigen Zugängen zwischen 1933 und 1945. Resümee aus der Sicht eines "Nach-Hörers" - 60 Tage nach der Live-Präsentation (Harald Albrecht)

\section{\#vBIB20 - die virtuelle Konferenz rund um bibliothekarische Themen}

Aufgrund der Auswirkungen der Covid-19-Pandemie musste der 109. Deutsche Bibliothekartag, der von 26. bis 29. Mai 2020 in Hannover stattfinden hätte sollen, abgesagt werden. Weil damit eine physische Zusammenkunft für Fortbildung sowie fachlichen und sozialen Austausch wie in den vergangenen Jahren nicht möglich war, entschlossen sich der Berufsverband Information Bibliothek (BIB) und die TIB - Leibniz-Informationszentrum Technik und Naturwissenschaften und Universitätsbibliothek, mit \#vBIB20 eine virtuelle Konferenz rund um bibliothekarische Themen durchzuführen.

Ute Engelkenmeier (BIB) und Sören Auer (TIB) wiesen in ihrem Geleitwort zur \#vBIB20 darauf hin, dass ein umfangreiches und vielfältiges Programm dadurch ermöglicht wurde, weil viele Einreichungen für das neue Veranstaltungsformat einlangten. An drei Tagen - von 26. bis 28. Mai 2020 - wurden über 100 Beiträge online in drei parallelen Sessions (virtuelle Räume) präsentiert und insgesamt fast 70 Stunden Programm in Form einer Online-Videokonferenz geboten. 
Die Themenpalette der Online-Vorträge reichte von der Agenda 2030 bis zum YouTube-Kanal für die Bibliothek. Im Rahmen der Tagung hatten auch traditionelle Bibliotheksthemen Platz: von der automatisierten Sacherschließung über Thesaurus, Terminologie und Ontologie bis zur GND, von Bibliotheksrecht über Bibliotheksstatistik, Kooperation von Fachinformationsdiensten, kooperative Langzeitarchivierung, Provenienzforschung, Urheberrechtspolitik bis zur Weiterbildung.

Neben den Themen Open Access - von Open Access-Workflows bis zum Open Access Monitor - und Forschungsdatenmanagement, denen jeweils mehrere Vorträge gewidmet waren, wurden auch bibliometrische Visualisierungen, die Bibliothek 4.0, Blockchain, künstliche Intelligenz und Trendmanagement in Bibliotheken behandelt. Ergänzt wurde das Programm um Vorträge zu gesellschaftspolitisch relevanten Themen, wie diversitätsorientiertes Personalmanagement in Bibliotheken, digitale Bibliotheksangebote in Haftanstalten und Rassismen in Bibliotheksbeständen. Der aktuellen Covid19-Thematik geschuldet waren Online-Vorträge zur Katastrophenplanung an Bibliotheken sowie zur internationalen Kooperation im Zeichen von Corona.

Die Fülle an Beiträgen trug wohl dazu bei, dass mehr als 3.000 Anmeldungen registriert wurden, sodass sich der Aufwand für die veranstaltenden Institutionen gelohnt hat. Eine Besonderheit von \#vBIB20 lag darin, dass sie von allen Interessierten kostenlos besucht werden konnte. Die Videos der Beiträge stehen über das AV-Portal der TIB zur Verfügung.

Sollte \#vBIB20 bedingt dadurch, dass Covid-19 noch längere Zeit größere physische Zusammenkünfte verunmöglicht oder aber dass sich die Form der Online-Konferenz als eigenständiges Angebot neben den klassischen Bibliothekartagen etabliert, eine Fortsetzung finden, wären folgende Verbesserungen wünschenswert:

- Zu einzelnen Themen wurden Vorträge zu verschiedenen Zeiten angeboten; wünschenswert wäre es, thematisch zusammenpassende Vorträge in einem gemeinsamen Vortragsblock zu platzieren.

- Aufgabe der Moderatorinnen und Moderatoren war es, im Anschluss an die jeweiligen Vorträge Fragen, die von Zuhörerinnen und Zuhörern mittels Chatfunktion gestellt wurden, in das Forum einzubringen, was aber aufgrund der begrenzten Zeit nur bedingt möglich war; es wäre spannend gewesen, sämtliche Kommentare für alle Teilnehmer*innen des jeweiligen Online-Vortrages transparent zu machen.

Davon unbenommen ist den Organisator*innen von \#vBIB20 großer Respekt dafür zu zollen, in so kurzer Zeit und bei problematischen Rah- 
menbedingungen - viele Bibliotheken befanden sich während der Zeit der virtuellen Konferenz in einer Phase zwischen Lockdown und dem Wiederhochfahren des Betriebes - eine hochkarätige Veranstaltung dieser Dimension bei einer sehr kurzen Vorbereitungszeit organisiert und professionell durchgeführt zu haben.

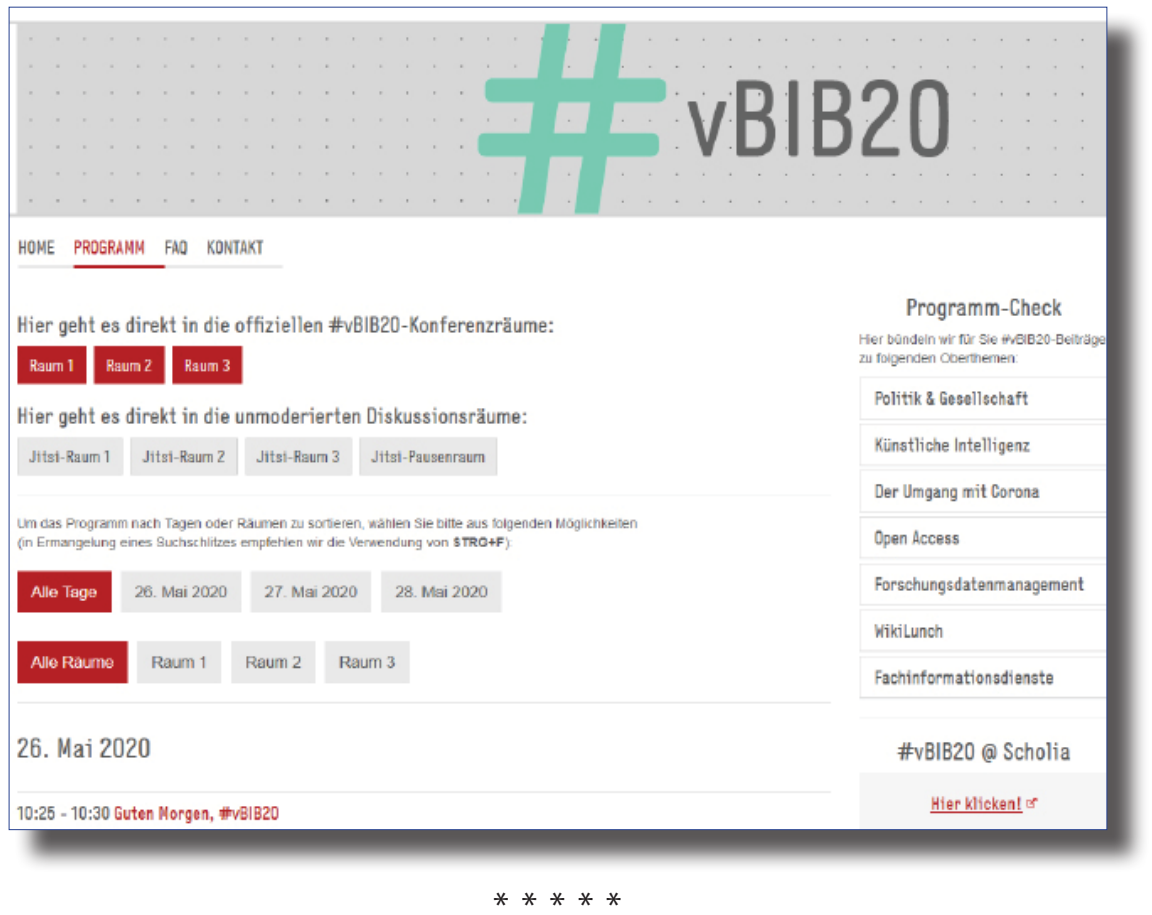

\section{Quo vadis Peer-Review?}

Bernhard Mittermaier (Zentralbibliothek des Forschungszentrum Jülich), bekannt für seine interessanten und kritischen Beiträge zum Publikationswesen, hat auch dieses Mal ein kontroverses Thema für seinen Vortrag gewählt: „Quo vadis Peer-Review“. Ziel von Peer-Review ist es, zu einer Verbesserung der Qualität der Publikation beizutragen, weil sowohl die Korrektheit der Methoden als auch die Richtigkeit der Aussagen validiert werden. Mit diesem Verfahren soll es zu einer Selbstregulierung innerhalb eines Fachgebiets kommen und auch eine gewisse Gatekeeper-Funktion erfüllt werden. 
In Mittermaiers Vortrag wurde jedoch aufgezeigt, dass Peer-Review bei genauerer Betrachtung viele Nachteile mit sich bringt und keinesfalls vor Fehlern oder Betrug schützt. Neben den hohen Kosten dieses Verfahrens kritisierte er vor allem die Verzögerung der Veröffentlichung, die Verhinderung innovativer Ideen und den möglichen Ideen-Diebstahl. Welche Alternativen gibt es also? Zu nennen sind Bibliometrie und Open Peer-Review. Ersteres betrachtete Mittermaier ebenfalls mit einiger Skepsis, da die Bibliometrie seiner Meinung nach nur in Einzelfällen, wie Evaluierungen oder bei der Erstellung von Rankings, als Ersatz dienen kann. Anders bewertete Mittermaier die Möglichkeit des Open Peer-Review. Er hält es für das ethisch überlegenere Verfahren, da man ein größeres Verantwortungsbewusstsein der Gutachter*innen, eine geringere Neigung zur Voreingenommenheit und eine geringere Gefahr des Datendiebstahls feststellen kann. Außerdem führe Open Peer-Review oftmals zu qualitativ besseren Gutachten. Bleibt abzuwarten, wie sich Review-Verfahren weiterentwickeln werden. Fest steht, dass es sich lohnt, sich kritisch mit dieser Thematik zu befassen.

Daniel Formanek

$* * * * *$

\section{Zurückgezogene Artikel in wissenschaftlichen Zeitschriften (Retractions): Ein Thema für wissenschaftliche Bibliotheken?!}

Jasmin Schmitz (ZB MED - Informationszentrum Lebenswissenschaften) führte per Webcam-Zuschaltung und PowerPoint Präsentation durch den Vortrag „Zurückgezogene Artikel in wissenschaftlichen Zeitschriften (Retractions): Ein Thema für wissenschaftliche Bibliotheken?!“. Der erste Teil der Präsentation befasste sich mit den Gründen für das Zurückziehen von wissenschaftlichen Artikeln und der Vorgehensweise zum Erwirken und Kennzeichnen von Retractions. Die Gründe lassen sich als festgestellte gröbere Fehler oder Verstöße gegen die gute wissenschaftliche Praxis zusammenfassen. Gilt ein Artikel als zurückgezogen, ist er mit einer Retraction Notice verknüpft. Schließlich kann der Artikel etwa gegebenenfalls ersetzt oder mit einem Wasserzeichen markiert werden. Für Bibliotheksmitarbeiter*innen interessant war vor allem der zweite Teil von Jasmin Schmitz' Vortrag, in dem es darum ging, wie man mit dem Thema Retractions umgehen und auch die Benutzer*innen darauf aufmerksam machen kann. Vorschläge waren zum Beispiel, bei Recherche-Schulungen auf Retractions einzugehen, zum kritischen Umgang mit wissenschaftlicher 
Literatur anzuregen und bei Fernleihen „Retraction Notices“ mitzuliefern. Auch nach Jasmin Schmitz' Präsentation konnten in einem Chat Fragen gestellt werden, bevor direkt anschließend die nächste Session eingeleitet wurde.

Caroline Reitbrecht

\section{DeepGreen im Testbetrieb: Erkenntnisse und Schlussfolgerungen}

Julia Boltze (Zuse Institute Berlin) und Regina Retter (Bayerische Staatsbibliothek) präsentierten in ihrem Online-Vortrag „DeepGreen im Testbetrieb: Erkenntnisse und Schlussfolgerungen" die gesammelten Erfahrungen der zweiten, erweiterten Testphase des Projekts DeepGreen. Das Ziel des Projekts, das 2016 ins Leben gerufen wurde und dessen Leitung der Kooperative Bibliotheksverbund Berlin-Brandenburg (KOBV) übernommen hat, ist die Etablierung eines automatisierten (und nicht mehr manuellen) Workflows zur Anreicherung von Open-Access-Repositorien mit Verlagsdaten. Somit soll die Open-Access-Zweitveröffentlichung von wissenschaftlichen Verlagspublikationen vereinfacht und beschleunigt werden. In Großbritannien ist es gelungen, aus einem ähnlichen Projekt, dem "Jisc publications router", ein dauerhaft angebotenes Service zu entwickeln. Getestet wurde die Drehscheibe DeepGreen von Oktober 2019 bis heute an 50 deutschen Bibliotheken als Betreiberinnen von Repositorien; fünf Verlage, darunter etwa SAGE Publications und BMJ, liefern als Partner Open-Access-Publikationen und Metadaten. Bisher wurden die Daten von 300.000 Open-Access-Artikeln auf diese Weise in Repositorien eingespeist. Für die Projektbeteiligten war natürlich das Feedback der Repositorienbetreiberinnen von besonderem Interesse. Ausgewertet und präsentiert wurden in einer PowerPoint-Präsentation Ergebnisse einer Online-Umfrage und eines Workshops:

- zum Umgang und der Verwendbarkeit der großen Datenlieferungen der Verlage

- zu Erfahrungen mit verschiedenen Repositoriensoftwaretypen

- zur Art von Anfragen und Notwendigkeit des Supports

Ein möglicher Grund, weshalb per DeepGreen gelieferte Artikel nicht online gestellt wurden, waren laut Umfrage beispielsweise Dubletten, die Daten waren bereits manuell eingegeben worden. Das Dublettenproblem sollte jedoch nur ein vorübergehendes sein und ist der erst recht kurzen Projektlaufzeit geschuldet. Obwohl die Konferenz \#vBIB20 aufgrund der Covid-19-Be- 
schränkungen „nur“ im virtuellen Raum stattfinden konnte und sowohl für Veranstalter*innen, Referent*innen und Teilnehmer*innen einige Herausforderungen vor allem technischer Art bot, gelang es den beiden Referentinnen, ihre Präsentation äußerst gut abgestimmt, informativ und spannend zu gestalten. Im Rahmen einer im Anschluss an den Vortrag angebotenen Fragerunde per Chat wurde die Möglichkeit, DeepGreen in Zukunft auch an österreichischen Bibliotheken zu testen, thematisiert. Bisher beschränkte sich das Angebot auf deutsche Institutionen, wobei eine Erweiterung des Projekts auf österreichische Bibliotheken für die Projektleitung grundsätzlich denkbar wäre. An dem Projekt DeepGreen Teilnehmende oder Interessierte können sich auf der Website https:// deepgreen.kobv.de über das Projekt, das noch bis Februar 2021 läuft, und dessen Fortschritt informieren.

Caroline Reitbrecht

$* * * * *$

\section{Kostentransparenz in OA-Transformationsverträgen}

Im Vortrag von Dirk Pieper (Universitätsbibliothek Bielefeld) zum Thema „Kostentransparenz in OA-Transformationsverträgen" ging es um die Forderung, Open Access Agreements transparent und vergleichbar zu machen. Hierfür war schon im Rahmen des DFG-Projekts INTACT (10/2015-09/2018) Open APC (Article Processing Charge) entwickelt worden (https://intactproject.org/openapc/). Open APC hat es sich zum Ziel gemacht, die Artikelkosten unter einer offenen Datenbanklizenz zu veröffentlichen, wodurch ein transparentes und nachnutzbares Reporting und Monitoring für wissenschaftliche Einrichtungen und Forschungsförderer ermöglicht wird. Zusätzlich dient die Aggregation von Kosten- und Publikationsdaten zur Unterstützung der Open-Access-Transformation. Laut Pieper wäre es möglich, ähnlich wie beim Springer Compact-Vertrag, mit Hilfe von Open APC die Wirkung des im Rahmen von DEAL abgeschlossenen Wiley-Vertrags auf die OA-Anteile von Wiley-Zeitschriften zu monitoren. Weiters sieht er, auf Basis der einrichtungsbezogenen Publikationszahlen und der Kostenzahlen, die Möglichkeit, ein intrainstitutionelles Kostenverteilungsmodell für künftige Transformationsverträge zu erstellen.

Daniel Formanek

$* * * * *$ 
Zum Themenbereich Leistungsmessung und Bibliotheksstatistik, moderiert von Bernhard Tempel (TIB - Leibniz-Informationszentrum Technik und Naturwissenschaften), gab es im Rahmen von \#vBIB20 am 27. Mai 2020 zwei Vorträge, die sich mit den aktuellen Neuerungen der DBS/OEBS, nämlich den neuen Personalkennzahlen und der Visitzählung, befassten.

\section{Die neuen Personalkennzahlen in der Deutschen Bibliotheksstatistik}

Ungeachtet der bereits vorgerückten Stunde und des eher trockenen Themas, wie der Vortragende Joachim Kreische (UB Dortmund) selbst bemerkte, fanden sich 160 Teilnehmer*innen virtuell in Raum 1 ein, um im Vortrag „Die neuen Personalkennzahlen in der Deutschen Bibliotheksstatistik Neue Perspektiven für den Leistungsvergleich?" zu hören, ob sich das neue Kennzahlenset als brauchbares Tool zum Leistungsvergleich denn nun bewährt hätte.

Seit 2019 gibt es innerhalb der Deutschen Bibliotheksstatistik (DBS) bzw. der Österreichischen Bibliotheksstatistik (OEBS) eine Erweiterung der Personalkennzahlen für wissenschaftliche Bibliotheken, bei der im Gegensatz zur bisherigen ausbildungsmäßigen Differenzierung zusätzlich auch die funktionsbezogenen Stellenanteile des Personals angegeben werden können. Die Teilnahme an diesem Kennzahlenblock innerhalb der DBS/OEBS ist freiwillig, die ermittelten Werte sind nur für die teilnehmenden Bibliotheken dieses gesonderten Fragebogenteils sichtbar (https://service-wiki.hbz-nrw.de/ display/DBS/Anleitung+erweiterte+Personalkennzahlerhebung).

26 Wissenschaftliche Bibliotheken nahmen 2019 dieses Angebot an, davon 18 Universitätsbibliotheken, zwei davon aus Österreich. Kreische, der auch Vorsitzender der Steuergruppe für Wissenschaftliche Bibliotheken ist, zeigte zwar Verständnis dafür, dass die Aufgabe, die Funktionsbereiche in einem $100 \%$-Verteilungsschlüssel abzubilden, durch die Verschiedenartigkeit der einzelnen Bibliotheken sicherlich manchmal schwierig sein könne, aber für die Aussagequalität absolut notwendig sei. Die vorgegebenen, sperrigen Funktionsbereiche wären dem Versuch einer Balance zwischen einem möglichst geringen Arbeitsaufwand und einem validen, differenzierten Datenoutput geschuldet.

Das sehr kleine Teilnehmerfeld der 26 Bibliotheken (etwa 10\% der DBS/OEBS-Bibliotheken) hat sich als relativ homogene Gruppe erwiesen, sodass sich aus der Sicht des Vortragenden der Vergleich der Personalkennzahlen für seine Bibliothek durchaus brauchbare Erkenntnisse ergaben. Generell gelte, dass beim Vergleichen von Bibliotheken vor allem die richtigen Fragen gestellt werden müssten, betonte Kreische, nämlich nicht 
„Bin ich besser oder schlechter?", sondern „Was will ich als Bibliothek? Wo liegen meine angestrebten Schwerpunkte?". Erst vor dem Hintergrund der strategischen Ziele würde eine Abweichung aussagekräftig. Sie kann nämlich erwartet oder gewollt sein, aber auch überraschen und zu einer Verbesserung führen, in unserem Fall zu einer Umlenkung von Personalressourcen.

Am Ende stellte Joachim Kreische aber relativierend fest, dass Personal-einsatzvergleiche derzeit keine Priorität hätten. Bibliotheken hätten vielmehr im Augenblick alle Hände voll zu tun, sich zukunftsfit zu machen und seien mit strategischen Umorientierungen und Neuaufstellungen beschäftigt. Das Personal solle die „innovativen Dinge“ tun, sodass die neuen Personalkennzahlen derzeit eher als Nischenprodukt gesehen werden können.

Elisabeth Erasimus

$* * * * *$

\section{Bibliotheksstatistik: Wie die Visitzählung in der DBS funktioniert und wie sie den Blick auf Bibliotheken verändert}

Der Vortrag zum Thema „Bibliotheksstatistik: Wie die Visitzählung in der DBS funktioniert und wie sie den Blick auf Bibliotheken verändert" wurde als Videoeinspielung von Gabriele Heugen-Ecker und Therese Nap (beide Hochschulbibliothekszentrum des Landes Nordrhein-Westfalen Köln) gemeinsam bestritten (https://av.tib.eu/media/47079).

Nach der Durststrecke der letzten Jahre (2015 wurde die Kooperationsvereinbarung zur Erhebung der virtuellen Nutzung mit der Hochschule für Medien in Stuttgart gelöst) gibt es seit 2019 wieder die Möglichkeit, die Zahl der virtuellen Besuche in die Bibliotheksstatistik einfließen zu lassen. Der neue Partner für die Visitzählung der Deutschen Bibliotheksstatistik (DBS) ist INFOnline, eine im Bereich Digital-Audience-Measurement erprobte Firma mit Sitz in Bonn.

Die Bedeutung einer Kennzahl für virtuelle Besuchen ist unbestritten und als Pendant zur Kennzahl der physischen Besuche für die Darstellung der Gesamtnutzung notwendig. Gabriele Heugen-Ecker und Therese Nap bewarben in ihrem Vortrag das neue Angebot der Visitzählung, das - zumindest in der Basiserhebung - kostenfrei, mit wenig Arbeitsaufwand verbunden und datenschutzrechtlich ordnungsgemäß geprüft wäre - nicht zu vergessen die 24-Stunden-Überwachung und der 
Realtime-Zugriff. Der Weg zur Visitzählung erfolgt für interessierte Bibliotheken in drei Schritten: Nach der Anmeldung (1) in einem zweistufigen Verfahren über die DBS und dann bei INFOnline wird in der Folge mit der Firma INFOnline ein Vertrag (2) abgeschlossen und schließlich mit dem Einbau (3) der erforderlichen Tags in die Bibliotheksseiten die Zählung gestartet (https://service-wiki.hbz-nrw.de/pages/viewpage. action? pageld=372998166).

Die Ergebnisse der Pilotphase 2019 zeigen, dass nur 12 von 357 DBSBibliotheken bereits aktiv gezählt haben (fehlende Daten wurden hochgerechnet). Im Juni 2019 startete die Anmeldung und am Ende des Jahres waren 62 Bibliotheken für die Visitzählung zwar angemeldet, aber mit dem nächsten Schritt, der Vertragnahme, wurde dann offenbar (noch) zugewartet. 19 Bibliotheken hatten Ende 2019 zwar immerhin bereits einen gültigen Vertrag mit der Firma INFOnline, aber noch keine Zählung aktiviert. Diese zögerliche Resonanz der Pilotzählung 2019 hat sehr überrascht. Um die Ursachen für diese Zurückhaltung herauszufinden, wurden die Bibliotheken, die sich zwar angemeldet, aber keinen Vertrag mit INFOnline abgeschlossen hatten bzw. die zwar schon einen Vertrag hatten, aber den Code (noch) nicht eingebaut hatten, in Form einer Umfrage um Feedback gebeten. Es stellte sich heraus, dass vor allem fehlende technische Unterstützung vor Ort und datenschutzrechtliche Probleme die zügige Realisierung der Visitzählung blockierten.

Obwohl sich für 2020 mit Stand März 2020 bereits ein starker Zuwachs von 74 angemeldeten Bibliotheken, 22 Vertragsinhaber*innen und 7 aktiv zählenden Bibliotheken abzeichnet, dürfen sich interessierte Bibliotheken von Seiten der DBS/OEBS eine kontinuierliche Unterstützung bei der Visitzählung sowie weitere technische Verbesserungen, durch die datenschutzrechtliche Bedenken ausgeräumt werden sollen, erwarten.

Elisabeth Erasimus

$* * * * *$

Forschungsdatenmanagement gestern, heute und morgen zwischen FAIR, CARE und EOSC. Ein Erfahrungsbericht mit einem digitalen Vortrag im Rahmen der virtuellen Konferenz \#vBIB20

Nachdem wir uns heuer damit abfinden mussten, dass keine öffentlichen Veranstaltungen möglich waren, vor allem nicht in der Größenordnung eines Deutschen Bibliothekartags, freute mich die Anfrage sehr, meinen 
geplanten Vortrag über unsere Erfahrungen mit dem Forschungsdatenmanagement an der Universitätsbibliothek Wien, am 28. Mai 2020 online im Rahmen der \#vbib20 abhalten zu dürfen, zu der sich schließlich mehr als 3.000 Personen angemeldet haben. Wer die Bibliothekartage mit den zahlreichen Parallelsessions, Arbeitssitzungen, Workshops usw. kennt, wird sich fragen, wie es möglich sein soll, so eine riesige Veranstaltung in den virtuellen Raum zu verlegen. Das erfordert eine penible Organisation und sehr viel Aufwand, immerhin gab es an drei Tagen über 100 Beiträge. Die Veranstalter*innen versorgten die Referent*innen und Moderator*innen vorab mit wichtigen Informationen und es wurde allen an mehreren Terminen eine Einführung in das verwendete Tool angeboten, sowie die Möglichkeit zu testen, wie man beispielsweise die eigenen Folien teilt, bzw. wie der Chat funktioniert. Es war für alle Beteiligten eine neue Situation, deshalb war auch eine gewisse Aufbruchsstimmung bei diesem Testlauf, der sehr gut funktioniert hat, zu erkennen. Natürlich passieren auch hin und wieder Fehler, man muss beispielsweise wissen, dass man als „Presenter“, wenn man das Meeting vorzeitig verlässt, keinesfalls „Meeting beenden“ anklicken darf. Ein falscher Klick und mehrere hundert Personen sind hinausgek(I)ickt. Letztendlich hat bei meinem Vortrag alles sehr gut funktioniert, es waren ca. 280 Personen im virtuellen Raum, die anschließend im Chat zahlreiche interessierte Fragen gestellt haben, aber es ist wieder mal deutlich geworden, dass persönliche Kommunikation schwer oder gar nicht ersetzbar ist. Warum, möchte ich anhand einiger Punkte erläutern:

\section{Alleine vor dem Bildschirm}

Man redet 20 Minuten gegen den eigenen Bildschirm ohne die Reaktionen der Zuhörer*innen einschätzen zu können. Ist das Publikum noch interessiert, wird getuschelt, verlassen Personen den Raum, spürt man eine gewisse Langeweile aufkommen? All diese Zeichen, die man oft unbewusst empfängt, wenn man am Rednerpult steht, fehlen. Somit ist man wie eingekapselt im eigenen Vortrag. Zwischendurch sorgt man sich noch, ob die Folien angezeigt werden und man gut zu hören ist.

\section{Fragen der Zuhörer*innen}

Wie groß das Interesse am Thema ist, lässt sich bei Vorträgen oft auch anhand der Fragen erkennen. Die Fragen wurden diesmal online per Chat gestellt, die die Vortragenden jedoch nicht sahen, sondern die ihnen von den jeweiligen Moderator*innen am Ende vorgelesen wurden. Auch hier 
fehlt der persönliche Kontakt. Man weiß letztendlich nicht, wer gefragt hat und ob die Antworten zur Klärung beigetragen haben.

\section{Keine Gespräche auf den Gängen}

All die belebenden Gespräche mit zufällig getroffenen Bekannten oder auch Diskussionen mit Personen, die man eben gemeinsam an einem Tisch stehend kennengelernt hat, fallen weg. Es gibt auch keine kurzen Besprechungen mit den eigenen Kolleg*innen, die im Alltag oft zu kurz kommen.

\section{Kein Eintauchen in ein Thema}

Stadtspaziergänge, Bibliotheksbesuche, Besuche bei den Aussteller*innen, alles fehlt. Das Eintauchen in die unglaublich vielfältige Bibliothekswelt, die Begegnungen mit Themen, mit denen man sonst kaum etwas zu tun hat, gibt es nicht. Es bleibt nur - wenn man sehr diszipliniert ist - das Fokussieren auf einzelne Vorträge.

\section{Keine Zufälligkeiten}

Der schöne und beflügelnde Ausdruck „Serendipity“, die zufälligen Entdeckungen, fehlen. Es kommt doch oft vor, dass bei einem noch so ausgeklügelten selbst ausgeheckten Veranstaltungsplan mit streng eingeteilten Vorträgen, in die man sich unbedingt setzen möchte, doch mal eine nette Kollegin oder ein netter Kollege woanders hinstrebt, man ihr oder ihm folgt und dafür mit einem interessanten Thema belohnt wird, das man davor noch gar nicht wahrgenommen hat. Digital muss man selbst auswählen, für Zufälligkeiten ist kein Platz.

\section{Zeiteffizienz}

Natürlich ist es finanziell und zeitlich effizienter zu Hause am Computer zu sitzen als eine Dienstreise zu buchen und mindestens drei Tage unterwegs zu sein, aber was geht uns dabei verloren? Informeller Austausch, das Sammeln von Ideen, das Networking, das gemeinsame Pläneschmieden...

\section{Die guten Seiten}

Allerdings gibt es auch positive Effekte bei digital abgehaltenen Veranstaltungen. Es haben auch Personen Zugang zu für sie wichtigen Informatio- 
nen, die vielleicht aus persönlichen oder aus dienstlichen Gründen keine Dienstreisen unternehmen können. Die Vorträge werden meist aufgezeichnet und sind, wenn man zur Vortragszeit verhindert ist, nachzuhören, bzw. bleiben die Folien abrufbar. Noch etwas soll nicht verschwiegen werden: Wenn man es in einen digitalen Raum geschafft hat, ist man meist fokussierter auf den Vortrag als in einem großen Raum. Man hat die Folien groß und deutlich vor sich und ist weder durch Umgebungsgeräusche noch durch die Kolleg*innen, die man eben im Raum entdeckt hat und begrüßen möchte, abgelenkt. Man verplaudert sich auch nicht am Gang oder vergisst die Zeit im angrenzenden Park.

\section{Ein großes Dankeschön}

An dieser Stelle übrigens herzlichen Dank an die Veranstalter*innen und alle Mitwirkenden, die sehr kooperativ und hilfsbereit waren und mit der digitalen Version des Bibliothekartags das Beste aus der Situation gemacht, den Vortragenden eine Chance für die Verbreitung ihrer Inhalte gegeben und das Entstehen einer große Informationslücke verhindert haben.

\section{Zum eigentlichen Vortrag}

Ziel des Vortrags war es, Erfahrungen mit Forschungsdatenmanagement an der Universitätsbibliothek Wien mit anderen zu teilen, unsere Entwicklungen und Strategien zu zeigen und auch auf die unterschiedlichen Handlungsfelder hinzuweisen, die uns nach und nach zugewachsen sind und die sich in den letzten Jahren stark erweitert haben.

Die Folien sind unter https://phaidra.univie.ac.at/o:1083994 abrufbar.

Susanne Blumesberger

$* * * * *$

\section{Resümee aus der Sicht eines Teilnehmers der virtuellen Konferenz \#vBIB20}

Die Initiative von BIB - Berufsverband Information Bibliothek e.V. und TIB - Leibniz-Informationszentrum Technik und Naturwissenschaften und Universitätsbibliothek, aufgrund der Covid-19-Pandemie eine virtuelle Konferenz \#vBIB20 zu veranstalten, hat sich als wirklich gute Idee und eine pro- 
bate Alternative zu bisherigen Konferenzen erwiesen. Die Umwelt wird es uns danken. Musste doch niemand mit Flugzeug oder Bahn anreisen. Jeder konnte teilnehmen. Nicht nur die wenigen arrivierten Mitarbeiter*innen, oder wie man so schön sagt: „Eh immer die Gleichen“ konnten hier teilnehmen, sondern jede ${ }^{*} r$ Mitarbeiter* in konnte, wenn er/sie sich die Zeit nehmen wollte, dabei sein.

Aber kann eine virtuelle Konferenz wirklich eine herkömmliche ersetzen? - Nicht gänzlich. Die Veranstalter*innen haben zwar alles getan, um einen virtuellen Ablauf zu gestalten, der jenen von herkömmlichen Veranstaltungen sehr nahekommt. So ganz kann es natürlich nicht gelingen. So gab es virtuelle Kaffee-Pausen nach den verschiedenen Panels, in denen man noch Fragen stellen und diskutieren konnte, doch nach 2-stündigem Head-Set-Tragen und durchgehendem Vortrag ist man nun aber doch froh, endlich mal eine wirkliche Pause einzulegen.

Man könnte nun meinen, dass man zukünftig die Kosten für Dienstreisen sparen kann, da man auch so an relevante Informationen kommt und sich einbringen kann. Wenn man dies nur auf die Vorträge bezieht, mag das ja vielleicht sogar stimmen. Doch jede*$r$, der/die regelmäßig an Tagungen im In- bzw. Ausland teilnimmt, weiß, dass Vieles informell stattfindet. Die interessantesten Diskussionen entstanden bei zufälligen Zusammentreffen beim Mittagessen, wo es nie genug Tische für alle gibt und sich immer verschiedenste Personen zusammenfinden und so plötzlich ins Gespräch kommen. Hier werden E-Mail-Adressen ausgetauscht, diskutiert, Bekanntschaften und ja sogar manchmal Freundschaften geknüpft. Jede ${ }^{*} r$ kennt diese Situationen.

So wie jede* $r$ weiß, dass ein offizieller Vortrag eben bloß ein offizieller Vortrag ist. Hier werden gewisse Probleme, die es vielleicht gab, nicht genau ausgeführt. Diese Dinge kommen oft nicht einmal bei den offiziellen Fragen im Anschluss an einen Vortrag aufs Tapet. Nein, dafür bedarf es der kleinen Kaffee-Pausen, der informellen Gespräche und Abendveranstaltungen. Dieser informelle Rahmen, der bei virtuellen Konferenzen fehlt, stellt aber einen nicht zu vernachlässigenden Mehrwehrt dar. Hier geht es nicht um „Social Distancing“, sondern um „Socialising“ oder wie es so schön auf Konferenzen heißt - „Come together“. 


\section{Provenienzforschung in der ZBW: Erste Ergebnisse zu verdächtigen Zu- gängen zwischen 1933 und 1945. Resümee aus der Sicht eines „Nach- Hörers" - 60 Tage nach der Live-Präsentation}

Monika Zarnitz und Norman Köhler präsentierten in ihrem Vortrag das NSProvenienzforschungsprojekt der ZBW Leibnitz-Informationszentrum Wissenschaft. Nach einem kurzen Eingangsstatement, das die Aufgaben und Ziele der NS-Provenienzforschung absteckte, lieferte Monika Zarnitz einen gerafften historischen Abriss zur Geschichte der ZBW und ihrer Vorgängerorganisationen, um den Teilnehmer*innen eine bessere Kontextualisierung ihres Forschungsgegenstandes zu ermöglichen. Danach präsentierte sie die Rahmenbedingungen und Ziele des mittlerweile seit 2018 laufenden Projektes, das vom Deutschen Zentrum Kulturgutverluste finanziert wird. Nach diesem ersten etwas kürzeren Teil des Vortrags stellte Norman Köhler das eigentliche Projekt näher dar. Er ging dabei auf die verschiedenen Projektphasen an den beiden Standorten Kiel und Hamburg, sowie auf noch geplante Projektphasen ein und gab am Ende noch einen kleinen Ausblick. Der Vortrag war ein sehr gelungener Werkstattbericht, der neben sehr interessanten Beispielen auch die Ergebnisse aus dem ersten sowie dem zweiten Projektjahr enthielt.

Die Idee, aufgrund der Covid-19-Pandemie eine virtuelle Konferenz \#vBIB20 zu veranstalten, halte ich grundsätzlich für sehr begrüßenswert und in der aktuellen Situation für sehr gelungen. Allerdings hoffe ich, dass reale Konferenzen und Tagungen in einer Post-Covid-19-Zeit nicht von solchen Formaten ersetzt werden. Reale Tagungen bieten einfach einige Vorteile, die eine Onlinekonferenz nicht oder nur eingeschränkt erfüllen kann. Ich konnte aus terminlichen Gründen nicht live an der Konferenz teilnehmen und hatte große Mühe Zeit zu finden, die Vorträge der virtuellen Konferenz \#vBIB20 nachzuhören, da im Tagesgeschäft immer „etwas Wichtigeres“ dazwischen kommt ... bei einer realen Konferenz (mit all ihren Mühseligkeiten - Anreise, Hotel,...) hat man viel mehr Gelegenheit sich auf die Veranstaltung einzulassen - man ist einfach nicht in seinem Büro und für die Kolleg*innen nicht so leicht greifbar. Dazu kommt, dass man meiner Erfahrung nach bei Konferenzen oft die interessantesten Informationen, Anregungen, Inputs, Ideen,... in den Pausen, beim Abendessen, etc. bekommt. Dazu kommt, dass analoges Networking immer besser funktioniert - ein kurzes reales Treffen ist oft effektiver als hunderte Emails. Trotzdem fände ich es gut, wenn auch in einer Post-Covid-19-Zeit bei Konferenzen die einzelnen Vorträge und Diskussionen aufgezeichnet würden, wie das in vielen Universitäten bei Präsenzphasen von Fernstudiengängen heute schon der Fall ist.

Harald Albrecht 
Harald Albrecht, BA

ORCID: https://orcid.org/0000-0002-9543-4172 Medizinische Universität Wien, Universitätsbibliothek E-Mail: harald.albrecht@meduniwien.ac.at

Mag. Bruno Bauer ORCID: http://orcid.org/0000-0002-4729-331X Medizinische Universität Wien, Universitätsbibliothek E-Mail: bruno.bauer@meduniwien.ac.at

Dr. ${ }^{\text {in }}$ Susanne Blumesberger ORCID: http://orcid.org/0000-0001-9018-623X Universität Wien, Bibliotheks- und Archivwesen E-Mail: susanne.blumesberger@univie.ac.at

Mag. ${ }^{a}$ Elisabeth Erasimus ORCID: https://orcid.org/0000-0001-6145-5074 Medizinische Universität Wien, Universitätsbibliothek E-Mail: elisabeth.erasimus@meduniwien.ac.at

Mag. Daniel Formanek

ORCID: https://orcid.org/0000-0002-0269-8022 Medizinische Universität Wien, Universitätsbibliothek E-Mail: daniel.formanek@meduniwien.ac.at

Mag. ${ }^{a}$ Caroline Reitbrecht ORCID: https://orcid.org/0000-0003-4141-2573 Medizinische Universität Wien, Universitätsbibliothek E-Mail: caroline.reitbrecht@meduniwien.ac.at 